\title{
How Talking Spreads Viruses
}

\author{
Speech can spray more saliva than sneezing or coughing, a finding that \\ impacts the measures people might take to avoid infection.
}

By Katherine Wright

W hen COVID-19 hit, it quickly became apparent that many people were catching the virus by breathing in contaminated air. That finding led governments to issue guidelines recommending that people stay at least two meters (six feet) apart and limit indoor gatherings. At the APS Division of Fluid Dynamics (DFD) conference two weeks ago, physicists studying viral transmission through speech presented their latest results. Their findings indicate that saliva sprayed while speaking could contribute significantly to the spread of the virus-more so than coughs or sneezes-and that people might want to step even further back from their interlocutors.

Sneeze, and about 10,000 drops fly out of your nose and mouth. Greet a friend with a quick "good morning," and that figure plummets to a few hundred. The emission from speech might sound small, but, unlike sneezes, words rarely occur in isolation. "Within about two to twenty seconds of speaking you

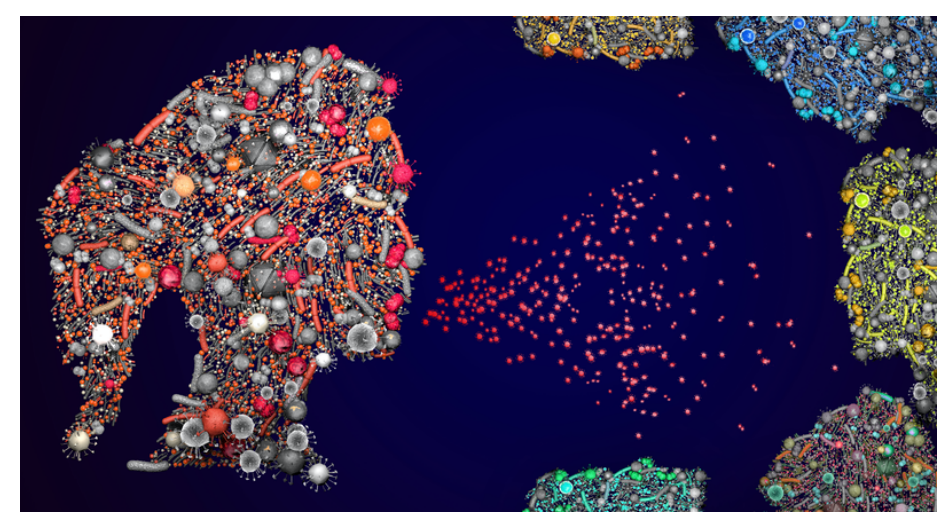

Saliva sprayed while speaking could contribute significantly to the spread of COVID-19, according to results reported at a recent conference.

Credit: Christoph Burgstedt/stock.adobe.com can produce as many [drops] as in a single cough," said Zu Puayen Tan of the National Chiao Tung University in Taiwan.

In his talk, Tan discussed the results of experiments that he and his collaborators performed, using high-speed imaging to track the aerosol plume of gas and drops that a human volunteer sprayed out when speaking different words with varied loudness. He found that syllables with hard consonants, such as ti, pa, and da, emitted larger and longer-lasting plumes than softer words, such as ma or sa (see How Speaking Creates Droplets That May Spread COVID-19).

"People might not think that they can pass on COVID-19 just by speaking," Tan said, but his data showed that speech-induced plumes can reach distances of $1.3 \mathrm{~m}$ within $2 \mathrm{~s}$, similar to those of coughs. Experiments presented by Lydia Bourouiba of the Massachusetts Institute of Technology (MIT), which recorded drops over longer distances and timescales, showed plumes that reached even further-up to $8 \mathrm{~m}$ ( $26 \mathrm{ft})$, a distance far beyond the separation recommended in physical-distance guidelines.

The 2-m social-distancing figure comes from experiments and theory that consider the motion of isolated drops moving though still air, said Bourouiba. But saliva is a complex spray, transported by gas from the lungs. This gas traps the drops and changes the evaporation conditions, something that "leads to completely different predictions of distance," she said.

Bourouiba's theories, which account for the accompanying gas, predict the fourfold longer distances that she sees in experiments.

Another factor that the 2-m rule fails to account for is the surfing of virus-containing saliva drops on ventilation airflows within rooms and buildings. This surfing can explain superspreader 


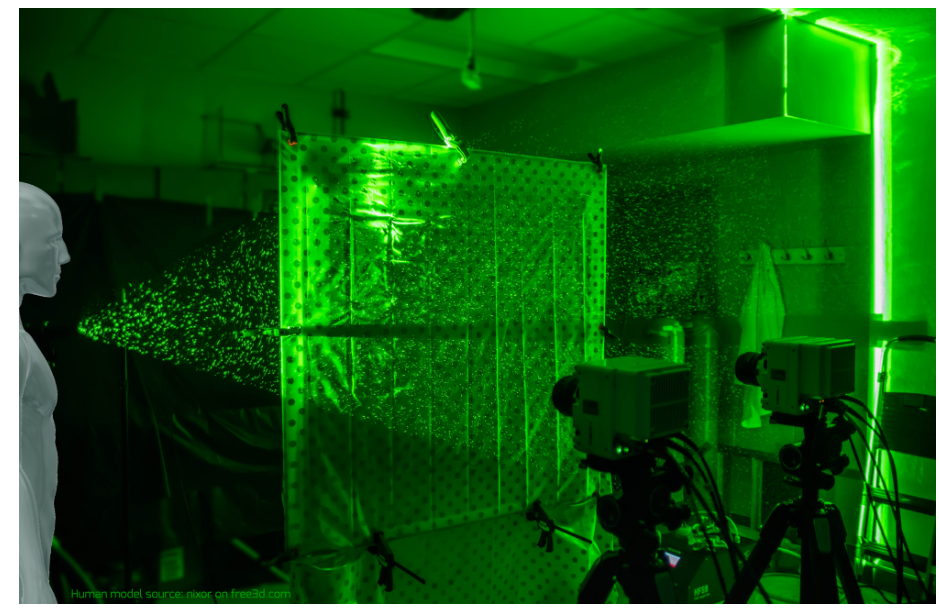

A photograph showing the experimental setup used by Tan and colleagues at Auburn University, Alabama, to study saliva spray released during coughing and speaking. The particles in the photos are small helium bubbles that were used to track the plume and are not the aerosol drops. Note that because of regulations in human subject research, the person has been overlaid with a graphic in the image.

Credit: Z. P. Tan/National Chiao Tung University

events, such as the one that occurred at a two-hour rehearsal in March of the Skagit Valley Chorale in Washington, where 53 people out of 61 caught COVID-19. The singers were spread out across a room roughly the size of a volleyball court. There is "overwhelming evidence" that the ventilation was to blame, said Martin Bazant from MIT. "The singers were mostly never in close contact."

Bazant has developed a model that calculates the risk of transmission in a given space by accounting for the rate of ventilation. His model shows that transport of virus-containing drops on ventilation-induced airflows can quickly contaminate a room, making the 2 - $m$ rule moot.

So, is there a way for people to gather safely indoors, especially during the holiday season? To help everyone better assess the risk of COVID-19 transmission, Bazant and his colleagues have turned their model into an online app that calculates the safe time and occupancy limits for any indoor space. (Bazant offers a free online course where people can learn the science behind the app.) Inputting the details of my dining room, for example, which is roughly $37 \mathrm{~m}^{2}\left(400 \mathrm{ft}^{2}\right)$ and has no active ventilation system in winter, 10 cotton-mask-wearing guests and I would be safe from infecting each other for 2.5 hours-assuming one of us had COVID-19, and we were all talking at a normal level. Opening the windows roughly doubles the safe time.

We could enjoy each other's company for longer-and still stay well-by donning N95 masks, specialized masks that keep 95\% of all airborne particles, including drops, from entering the lungs. These masks are typically worn by clinicians and were sold out quickly after the pandemic hit. But Nicole Bustos, a graduate student with Bourouiba, presented a fix for that problem. She showed that a homemade mask consisting of a coffee filter sandwiched between two layers of cotton from a t-shirt was just as effective as an N95 mask.

Katherine Wright is a Senior Editor for Physics. 